\title{
SOFTWARE Y HERRAMIENTAS ESPECIALIZADAS EN BENEFICIO DE LA DOCENCIA
}

\author{
Gustavo Anabalón *
}

* Director, Dirección de Servicios de Tecnologías de Información (STI), Universidad de Chile. 


\section{RESUMEN}

El fuerte impacto de las tecnologías de información y comunicaciones (TICs) en prácticamente todas las áreas de desarrollo de nuestra sociedad se ha manifestado con la apertura de grandes oportunidades para mejorar la efectividad y productividad aplicables a las más variadas disciplinas. En la primera parte de este documento se describen los principales factores que han condicionado la incorporación de la tecnología al desarrollo de la educación, para luego demarcar el amplio espectro de necesidades académicas y de la docencia propiamente tal, donde los distintos programas y aplicaciones computacionales pueden aportar significativamente, así como las diversas opciones de licenciamiento que ofrece el mercado. A continuación se mencionan algunos aspectos de la experiencia de la Universidad de Chile, se describen algunos acuerdos y convenios de licenciamiento, se reseñan algunas prácticas en el uso de plataformas y programas de autor y se señalan algunos productos creados con desarrollos propios. Finalmente, se reflexiona sobre algunos desafíos institucionales en torno al tema.

\section{ABSTRACT}

The strong impact of the information and communication technologies on almost all our society's developing areas has opened unexpected opportunities for productivity's improvement. To begin with, this paper describes the determining elements that have influenced on including technology in education. Secondly, it points out the academic and teaching needs which can be assisted by computational programs, as well as the various degrees in the market. It continues to point out the experience inside the University of Chile, its agreements, some author's platforms and programs, and its own products. Finally, it talks about some of the institutional challenges on the subject. 


\section{SOFTWARE Y HERRAMIENTAS ESPECIALIZADAS EN BENEFICIO DE LA DOCENCIA}

\section{CONTEXTO GENERAL}

El impresionante desarrollo de la tecnología ha producido un salto fundamental en la productividad de prácticamente todas las actividades de nuestra sociedad. Los logros en la medicina, la economía, la industria y otras áreas han sido fuertemente impulsados por dos elementos comunes: la informática y las comunicaciones.

El surgimiento de los computadores y, en particular, la invención del microprocesador -que dio la oportunidad de tener en el escritorio o en un bolsillo una capacidad de procesamiento que, hasta hace algunos años, estaba reservado para grandes centros de investigaciónhan permitido contar con herramientas simples y poderosas que, junto con facilitarnos nuestras tareas, nos obliga a llegar a niveles insospechados de producción y conocimiento.

El impacto ha sido fuerte; sin embargo, en el ámbito de la educación se perciben dos elementos que interactúan en forma dicotómica. Por una parte, han proliferado herramientas que facilitan los procesos rutinarios que no requieren del aporte de la creatividad o inteligencia humana y que constituyen un soporte a las metodologías clásicas de aprendizaje, permitiendo al alumno y al profesor concentrarse en la esencia de su materia más que en el desarrollo de habilidades mecánicas. No obstante, paralelamente, la oportunidad que da la tecnología de encontrar nuevas y mejores formas de obtener y transferir conocimiento no ha sido del todo explorada ni ha tenido el desarrollo esperado, al menos no con la velocidad que se ha visto en otros ámbitos. 
Algunos factores han condicionado este desarrollo. Por una parte, el ambiente que generan las limitantes ergonómicas de un computador. Por otra, el lento avance de las técnicas de programación y la carencia de principios psicológicos apropiados sobre el proceso de aprendizaje, aplicables al diseño instruccional. Si consideramos que la principal motivación para el desarrollo de la tecnología está dada por la constante lucha por un mejor posicionamiento competitivo de la industria, es allí donde deben esperarse los primeros logros; por el contrario, en el mercado de la educación siempre ha sido difícil acceder a recursos para la investigación y el desarrollo de metodologías y técnicas de enseñanza-aprendizaje y, peor aún, para el desarrollo de herramientas apropiadas que complementen y fortalezcan esas mismas metodologías. Finalmente, terminamos conformándonos con adaptar medios cuya génesis no necesariamente fue concebida para la educación ${ }^{1}$.

En este escenario, los primeros avances en el desarrollo de software educativo nacieron bajo el concepto de Instrucción Asistida por Computador (CAI), a fines de los años cincuenta. El CAI no era otra cosa que el desarrollo de tutoriales determinísticos que inducían al alumno a seguir un camino predeterminado ${ }^{2}$. Conforme disminuyeron los costos, y con ello el acceso a mejor tecnología, el concepto de CAI fue decayendo y aparecieron otros más globales hasta los que finalmente pueden identificarse como e-learning, es decir, aprendizaje electrónico (en su acepción más básica). Llegado a este punto se ha presentado la confusión de dos conceptos complementarios, pero esencialmente diferentes: el e-learning o conjunto de técnicas de enseñanza-aprendizaje asistidas por computador, y la educación a distancia, la cual, si bien se ve fortalecida con el desarrollo actual de las TICs, en sí misma es un concepto bastante antiguo relativo a la mediatización de la enseñanza-aprendizaje a través de cualquier tipo de soporte, inclusive los libros. Sobre la base de estos elementos, ha proliferado un sinnúmero de herramientas ${ }^{3}$ de administración y desarrollo de cursos a distancia, basados en e-learning, que permiten eliminar las barreras de tiempo y espacio entre alumnos y profesores.

1 Por ejemplo, la educación no ha explotado en todo su potencial el desarrollo de la tecnología de los juegos.

2 Estos modelos tenían una fuerte influencia conductista, es decir, enfocados en la repetición de patrones hasta que se realicen en forma automática.

3 Ver, por ejemplo, el sitio: http://e-learning.bankhacker.com/ 
En el contexto universitario, el software utilizado para fines de docencia no pasa, necesariamente, por la selección y uso de medios orientados, específicamente, a apoyar los procesos propios de la enseñanza, sino que engloba un espectro mucho más amplio, que va desde la utilización de aplicaciones de propósito general, como es un procesador de textos, hasta la de un complejo simulador, pasando por aquellos módulos de apoyo administrativo o bien la herramienta fundamental de una disciplina. La variedad de software que se requieren en una institución compleja como la Universidad de Chile es tan amplia, que para cada área del conocimiento es preciso encontrar algún producto específico. En el transcurso de este documento abordaremos los diferentes perfiles de programas computacionales, los que, de forma directa o indirecta, son aplicables a la docencia.

\section{SELECCIÓN DE SOFTWARE}

En el proceso de selección de herramientas como aplicaciones tecnológicas al ámbito académico y su utilización al servicio de diversos tipos de necesidades (educativas propiamente tales, de gestión académica o, sencillamente, software utilitario), instituciones como las universidades pueden optar, básicamente, por dos caminos: desarrollar internamente software especializado o adquirir paquetes estándares en el mercado. En mi opinión, la calidad del software está determinada no sólo por sus aspectos técnicos, sino que, además, por su flexibilidad para adaptarse a necesidades particulares y por los servicios complementarios que ofrece. Durante un proceso de evaluación previa, debe conjugarse el costo del desarrollo con el que significa cambiar las viejas prácticas cuando estas últimas están consolidadas y no hay razones claras que aseguren que la adopción de nuevos procesos, necesariamente, conlleve un mejoramiento o sea aplicable en la realidad local.

En el caso de las herramientas comerciales, es relativamente fácil evaluar sus funcionalidades, por lo general es posible conseguir licencias de prueba (trial) del software que, en la mayoría de los casos, se pueden obtener directamente en Internet.

En cuanto a la obtención del licenciamiento, existen diversas modalidades. Algunas ofrecen licencias de uso limitado en el tiempo 
que pueden renovarse periódicamente; otras cobran un valor por la licencia y, en forma separada, un contrato por servicios de soporte y actualización de versiones; o bien, un concepto que cada vez toma más fuerza, arriendan los servicios de aplicaciones, lo que significa disponer del uso del software sin necesidad de invertir en su administración ni en los servidores que lo contienen. Cada una de estas opciones tiene características singulares que las hacen más o menos atractivas, dependiendo de factores como necesidades de independencia, de intervención en la configuración de parámetros específicos o bien, de razones estratégicas, en el sentido de que, por ejemplo, no siempre es bien visto entregar a terceros la administración de recursos que pueden ser críticos. El software es un componente tecnológico que, en conjunto con otros de carácter más rígido y tangible como computadores, sistemas de comunicación o de almacenamiento, permite que esta misma infraestructura pueda tener múltiples usos y aplicaciones. En este contexto, podemos distinguir clases de software en función de las dimensiones que nos interese analizar.

Herramientas educativas y de apoyo a la cátedra

Dentro de éstas se cuentan los sistemas de biblioteca y los de apoyo infocomunicacional a la divulgación de contenido mediante Internet. Están incluidos también los simuladores y tutoriales, desarrollados especialmente para el aprendizaje de materias o disciplinas específicas.

Herramientas de escritorio, creación y desarrollo

Dan respuesta a las necesidades básicas de funcionamiento administrativo, estudio, preparación de material, análisis, cálculo, diseño y desarrollo. En este grupo encontramos la mayor variedad: procesadores de texto, hojas de cálculo, editores de video, audio, dibujo, lenguajes de programación, simuladores, y otros.

Herramientas de colaboración, comunicaciones y acceso a la información

Son instrumentos de coordinación que simplifican el trabajo en equipo en cualquier organización. Entre ellas se cuentan los sistemas de mensajería, de agenda, de comunicación sincrónica y de búsqueda y acceso a la información. 
Herramientas de plataforma

Sistemas básicos que permiten el sustento lógico de los servicios especializados y su protección, por ejemplo, sistemas operativos, servidores web, servidores de bases de datos, antivirus, y otros.

Sistemas de información administrativos

Encargados de apoyar los procesos administrativos de la organización, tanto al soporte de la actividad docente propiamente tal, como las tareas de carácter operacional y de gestión. Su rol es cada vez más relevante, puesto que su integración con las herramientas educativas y de apoyo a la cátedra permite vincular los aspectos académicos con los administrativos, entregando un servicio integral y eficiente, tanto a docentes como a estudiantes.

\section{EXPERIENCIA DE LA UNIVERSIDAD DE CHILE}

La continua integración e incorporación de las tecnologías de información constituye uno de los desafíos permanentes de la Universidad de Chile, para mantener su posición de liderazgo en el ámbito de la educación superior en nuestro país.

Si bien es cierto nuestra institución cuenta con académicos de gran prestigio y compromiso institucional, y con un estudiantado de alto potencial, existen debilidades de integración entre unidades académicas. Incorporar nuevas tecnologías de comunicación a los procesos docentes es un objetivo estratégico que se enmarca en la aspiración más amplia de consolidar la calidad y pertinencia de los programas de pregrado.

La universidad del futuro debe contar no solamente con infraestructura física y humana sino que, también, disponer de infraestructura tecnológica bajo la forma de recursos de software y, simultáneamente, del know how necesario para extraerles su mejor rendimiento.

En los últimos años, el desarrollo de las tecnologías de información y comunicación en la institución ha considerado, 
esencialmente, fortalecer una infraestructura tecnológica que opere bajo los criterios de multimedialidad y conectividad global, implementar y desarrollar programas de entrenamiento en el uso de esas tecnologías y emprender iniciativas enfocadas a la producción y adquisición de contenidos de información digital, como programas de educación interactiva, a distancia y servicios de bibliotecas digitales.

Paralelamente, avanzan en este sentido las unidades de innovación y desarrollo, radicadas en las propias facultades y que conocen más de cerca sus necesidades específicas. En este campo se ha respetado y promovido la autonomía de las unidades docentes, las que han invertido en programas educativos a distancia y en innovaciones tecnológicas para la docencia, ya sea en aspectos metodológicos, de apoyo a la cátedra o de acceso a la información.

En este proceso, las postulaciones a recursos del Fondo Competitivo del Programa Mecesup han privilegiado iniciativas que incorporen, crecientemente, tecnologías de información y comunicación al proceso docente con libre acceso de los estudiantes. Los recursos conseguidos han significado un importante impulso a esas actividades. Simultáneamente, se busca apoyar a la investigación y a los servicios académicos, ampliando el libre acceso a la información.

\section{Licenciamiento Corporativo}

Entre las herramientas tecnológicas de escritorio de uso más expandido, están las creadas por Microsoft, incorporadas en la suite Office; la Universidad de Chile ha firmado un convenio de licenciamiento global llamado Campus Agreement, que comprende a la institución toda y no máquinas individualizadas.

El principal beneficio consiste en que permite mantener controlado el licenciamiento a un costo considerablemente más bajo que el individual sin tener que invertir en esfuerzos de fiscalización interna y, adicionalmente, estar al día en las últimas versiones de cada software incorporado en el acuerdo y de los sistemas operativos de cada microcomputador de la universidad.

La política corporativa prevé que las facultades e institutos puedan integrar al contrato aplicaciones más especializadas según los requerimientos específicos de sus actividades académicas. 
Otro beneficio consiste en la posibilidad de acceder a las Academias Microsoft, lo que significa que las instituciones académicas podrán capacitar a los alumnos en las tecnologías del software. Este aspecto es relevante para la formación complementaria de los futuros profesionales porque les permitirá adquirir habilidades que son valoradas por el mercado, facilitando su futura inserción laboral.

También se torna posible que la institución educativa pueda realizar un proceso de transferencia tecnológica, dentro de lo que se establece un marco para el correcto uso, instalación y manutención de los productos Microsoft y del desarrollo de software basado en estas tecnologías.

Otra empresa con la que se ha llegado a un acuerdo de cooperación para el licenciamiento de software de la categoría escritorio y otros más específicos es Sun Microsystems. Si bien los productos que proporciona son de utilización menos expandida, su uso es más especializado y de carácter profesional. Algunos de los acuerdos con esta empresa comprenden acceso al sitio web de entrenamiento, donde profesionales de la universidad pueden acceder a capacitación en StarOffice, Sistema Operativo, Java y a la totalidad de aplicaciones de su software.

Por otra parte, en el marco de este convenio se situará un centro de entrenamiento orientado a alumnos de pregrado, quienes tendrán acceso a opciones de significativo valor. Certificaciones en tecnología Sun y entrenamiento de preparación para obtener la certificación Sylvan en dichas materias, como el Sistema Operativo Solaris y Java, son algunos de los beneficios que conlleva el acuerdo.

Plataformas y herramientas de apoyo

En el proceso de madurez de la incorporación de tecnologías de información, la Universidad de Chile se encuentra en una fase más centrada en la generación de contenidos de valor que en los de infraestructura.

Bajo este criterio, la educación a distancia y el e-learning cobran prioridad. La institución está en permanente evaluación de las 
tendencias actuales con el fin de integrar, oportunamente, desarrollos que signifiquen un real mejoramiento de la calidad de la docencia y una mayor efectividad de los procesos de aprendizaje.

En los últimos cuatro años han aparecido lenguajes de autor para la elaboración de materiales de instrucción; permiten que un diseñador o un experto en contenido se adapten a una determinada estructura y, siguiendo sencillas rutinas, elaboren materiales de enseñanza sin necesidad de dominar lenguajes de programación.

El concepto de "objetos", utilizado en técnicas de programación, puede inferirse en la educación aplicándolo a objetos de aprendizaje. En este sentido, se pueden desarrollar actividades educativas que se rotulen como objetos que serán reutilizados para otros cursos. Su mayor impacto es la aceleración del desarrollo de programas educativos al disponer de una amplia base de material pedagógico. Esta visión y la necesidad de contar con instrumentos que tengan un reconocimiento global obligan a incorporar plataformas capaces de realizar bien la tarea requerida y de integrar sus productos a estándares universales. Este criterio es una premisa gravitante cuando se evalúan herramientas corporativas que consideran tanto las de administración de contenido como aquellas de desarrollo de objetos educativos para la edición de videos, imágenes, sonidos e interacción con el alumno.

Web-CT y algo más que contenidos en la Red

El software que incorporó la universidad para la distribución de cursos a distancia y para fomentar el desarrollo de esta disciplina es Web$\mathrm{CT}^{4}{ }^{4}$ Consiste en un sistema de administración de cursos que permite la creación y su manejo vía computador; hoy en día es utilizado por gran cantidad de importantes universidades en el mundo, dada su flexibilidad y adaptabilidad a los distintos requerimientos. Permite administrar el sistema de cursos en Internet (perfil administrador), crear cursos (perfil docente) y aprender a través de la web (perfil del alumno).

4 Web-CT surge de un desarrollo propio de la Universidad de Alberta (Canadá). Cumple con las normas LTSC (Learning Technology Standards Committee) de IEEE: Norma ISO y ADL (Advanced Distributed Learning): modelo SCORM.

Desde el año 2002 la Universidad de Chile, a través de la Dirección de Servicios Tecnológicos de Información (STI), cuenta con licenciamiento corporativo. 
Una experiencia en el uso de esta herramienta ha sido el Programa de Educación Continua para el magisterio (PEC), que tiene como misión colaborar en el perfeccionamiento de profesores en ejercicio a lo largo de todo el país.

En una fase de evaluación previa sobre la metodología para abordar este proyecto, el PEC inició un proceso de reflexión para descubrir cómo poner los elementos multimediáticos al servicio del aprendizaje. Elementos como el audio, la imagen, el movimiento y la interactividad fueron considerados en el diseño del curso como elementos clave para ilustrar, agilizar, apoyar y motivar la construcción de conocimiento que el perfeccionamiento docente plantea. Recientemente, fruto de este proceso, se ha creado el primer curso interactivo en modalidad e-learning: "Convivencia escolar en la enseñanza media”. A través de múltiples actividades dinámicas de aprendizaje, y aprovechando las potencialidades de Web-CT en materia de herramientas de comunicación (tales como foros, chat), el alumno puede construir conocimiento de forma entretenida, didáctica y conectada.

Portales y administradores de contenido (Bea Web-Logic)

En otro ámbito, con recursos obtenidos a través de proyectos, la Universidad ha adquirido el software especializado Bea Web-Logic para la administración automatizada de contenidos a través de Internet. Con esta nueva plataforma se hace más eficiente la gestión de información institucional, ya que se podrá optimizar la producción de sitios web y ofrecer un servicio personalizado a los alumnos.

A través de Bea Web-Logic es posible desarrollar portales web según las características individuales del visitante. Este concepto se está incorporando al portal de Cursos de Formación General ${ }^{5}$ que ofrece el Departamento de Pregrado y al nuevo portal que tendrá la Universidad de Chile.

5 En el marco de la Reforma del Pregrado de la Universidad de Chile, el programa de Cursos de Formación General (CFG) se ofrece a los alumnos de todas las carreras con el objetivo de que éstos complementen su educación con materias de diversos ámbitos del conocimiento y, de esta forma, proveer de egresados más cultos y con una visión profesional más integral y multidisciplinaria. 
A estos servicios personalizados se suma la existencia de un directorio único de personas, lo cual contribuye al desarrollo de bases de datos con información restringida, se establece un acceso único a los portales y, además, se autentifica la información de cada usuario. ${ }^{6}$

\section{U- Cursos, recursos para apoyar a la cátedra}

El organismo con mayor experiencia en desarrollo tecnológico es la Facultad de Ciencias Físicas y Matemáticas, la cual, dentro del contexto de su plan estratégico de modernización y mejoramiento de su docencia, ha diseñado e implementado en una primera fase una innovadora herramienta infotecnológica, basada completamente en tecnología Internet, denominada U-Cursos, cuyo propósito es apoyar el desarrollo de la docencia presencial y facilitar la aplicación de nuevas y mejores metodologías de enseñanza-aprendizaje.

Consiste, básicamente, en un software que permite a los profesores crear en forma autónoma sitios web de aprendizaje para cada uno de los cursos que dictan. De manera muy sencilla, pueden subir material docente al sistema, crear foros, crear enlaces de interés, planificar actividades docentes a través de un calendario de actividades y agenda electrónica, obtener listas de cursos, administrar tareas, publicar notas y enviar correos electrónicos masivos, entre otras funcionalidades.

U-Cursos facilita una administración descentralizada del sistema ya que las decisiones respecto a lo que se puede publicar las toma el académico, quien mantiene su propia página de cátedra y ofrece otras características como crear y supervisar comunidades ligadas a la clase impartida.

Además, con mensajería SMS de telefonía celular, se puede mantener comunicación directa con los estudiantes respecto a día y

6 Este directorio recibe el nombre de LDAP (sigla en inglés), que se traduce como Protocolo de Acceso Ligero y permite conectar bases de datos con los distintos servicios web al sistema central. Contando con un solo directorio, la información se puede jerarquizar; además, es posible entregar privilegios según el usuario y acceder al correo electrónico personal, entre otros servicios.

Todo esto recurriendo a la misma base de datos, por lo que los tiempos de transferencia de información disminuyen y la operacionalización de requerimientos, a través de la web, se simplifica. Un ejemplo concreto de servicios que entrega la aplicación de Bea Web-Logic, en conjunto con LDAP, es que evita la multiplicidad de contraseñas. 
hora de algún foro de discusión, sala de la asignatura o una simple suspensión de actividades.

Luego de tres semestres de operación, U-Cursos cuenta con 6.000 usuarios entre alumnos y profesores de las Facultades de Ciencias Físicas y Matemáticas, Derecho y Medicina. Actualmente, atiende un promedio mensual de 80.000 sesiones de trabajo y se han publicado más de 8.000 archivos de material docente de los más variados temas.

U-Cursos requiere interactuar con un sistema de información curricular que le proporcione los datos base asociados a los estudiantes y a las asignaturas que estén cursando. Para ello, el sistema de información corporativo $\mathrm{GUIA}^{7}$ realiza la administración global de todos los aspectos relativos al alumno, tanto en el plano académico como en el socioeconómico y financiero.

Los desafíos son continuar su desarrollo incorporando mayores funcionalidades y extenderlo a toda la universidad, para así cubrir una población de 25.000 estudiantes. De esta manera, se están dando pasos importantes hacia la homogeneización de las herramientas institucionales y con ello apoyando los lineamientos estratégicos orientados hacia la transversalización de los programas de pregrado.

SAS, Sistema de análisis estadístico para aprendizaje especializado e investigación

El sistema de SAS provee la manera más eficiente de transformar los datos estadísticos en información, ya que datos y aplicaciones que corren en diferentes plataformas pueden ser integrados en un único ambiente de software $y$, de esta manera, entregar a los usuarios información relevante y actualizada. Permite un acceso transparente a cualquier fuente de datos, incluyendo archivos planos, jerárquicos y, los más importantes, manejadores de bases de datos relacionales. También incluye su propia base de datos de información para almacenar y manipular los datos, es decir, un DataWarehouse ${ }^{8}$.

7 Gestión Universitaria de Información del Alumno.

8 DataWarehouse: Sistema de almacenamiento que permite analizar grandes volúmenes de datos, tradicionalmente con objeto de medir aspectos relacionados con la gestión y resolver correlaciones entre diferentes variables de un sistema. 
El sistema permite un amplio rango de aplicaciones, destacándose el análisis estadístico, análisis gráfico de datos, análisis de datos guiado, mejoramiento de la calidad, diseño experimental, administración de proyectos, programación lineal y no lineal, generación de reportes y gráficas, manipulación y despliegue de imágenes, sistemas de información geográfico, visualización multidimensional de datos, aplicaciones de multimedia, así como los sistemas de información ejecutiva.

Los mayores usuarios de SAS son los investigadores; sin embargo, tiene muchas utilidades en la docencia, ya sea como herramienta de análisis estadístico en distintas disciplinas -que van desde la economía a la medicina-, como en el aprendizaje de las técnicas y métodos estadísticos propiamente tales.

\section{HERRAMIENTAS DE COMUNICACIÓN Y DE ACCESO A LA INFORMACIÓN}

\section{Sistema de bibliotecas}

El mejoramiento y desarrollo del acceso a contenido e información de bibliotecas, desde hace ya varios años, se han enfocado a la producción y adquisición de contenidos de información digital. Actualmente, más de 7.000 documentos se encuentran disponibles a través de múltiples servicios: Biblioteca digital de la Universidad de Chile ${ }^{9}$, que reúne en un solo sitio web libros, revistas, tesis, apuntes, e incorpora funcionalidades para que los académicos publiquen electrónicamente sus textos; sitio de revistas electrónicas, con alrededor de 5.000 revistas de texto completo y servicio Al Día ${ }^{10}$ con acceso a las tablas de contenidos de las más de 1.600 revistas especializadas; sitio

9 Ver sitio: http://www.bibliotecadigital.cl

10 Al Día es un sistema de alerta que permite a los académicos conocer, a través de Internet, las tablas de contenidos de las más de mil seiscientas revistas especializadas suscritas por nuestra universidad y acceder, de manera instantánea en la mayoría de los casos, a los textos completos de los últimos artículos publicados. Además, Al Día, en su variante Express, funciona como un servicio de alerta permanentemente actualizado, que informa -vía correo electrónico- las tablas de contenido del último número editado de las publicaciones definidas por el usuario y notifica sobre las nuevas revistas adquiridas por las bibliotecas de la universidad. Ver sitio: http://www.al-dia.cl/ 
Cybertesis ${ }^{11}$, que promueve la publicación y difusión de las tesis de los alumnos.

Apoyo a la administración y gestión académica

Hasta 1999 los sistemas operacionales de la Universidad de Chile no estaban preparados para satisfacer las necesidades institucionales. Debido a una administración descentralizada, así como a la ausencia de políticas y soluciones centrales efectivas de apoyo a la gestión, tanto académica como administrativa, estos sistemas existían de manera disociada, utilizando diferentes plataformas tecnológicas y, en general, modeladas exclusivamente para responder a procesos administrativos y requerimientos muy específicos.

Esta heterogeneidad produjo, entre otras dificultades: un alto grado de burocracia; escaso apoyo hacia la gestión universitaria; altos costo en tiempo y esfuerzo para obtener información; bajo nivel de seguridad, oportunidad y calidad de los procesos administrativos existentes; y servicios muy deficientes a los alumnos y académicos.

En este escenario, en el año 1999, con aportes del Fondo de Desarrollo Institucional de MINEDUC, se aprobó el desarrollo de un nuevo sistema de información corporativo, cuyos lineamientos contemplaron, en una visión de largo plazo, los siguientes componentes: diseño y permanente evolución del modelo de datos corporativo; construcción de los sistemas de información operacionales que den soporte a los procesos de administración universitaria y construcción de un sistema de información de apoyo a la toma de decisiones.

11 El sitio web de Cybertesis permite acceder a los textos completos de las tesis realizadas por los estudiantes de las diferentes carreras universitarias como requisito para la obtención de título o grado académico. La gran innovación de esta iniciativa es la integración de altos estándares en la producción y transferencia de textos electrónicos. SISIB ha invertido mucho esfuerzo en el estudio y mantención de una cadena de producción sobre la base del estándar SGML, que permite la estructuración de textos en un formato de conservación compatible y exportable a cualquier otro protocolo de comunicaciones.

Este proyecto es el resultado de un programa de cooperación entre la Universidad de Montreal, la Universidad de Lyon y nuestra casa de estudios, con el apoyo de Unesco. Ver sitio: http:/ /www.cybertesis.cl 
Modelo corporativo de datos

Privilegia la visión global por sobre la atención a requerimientos individuales. En él están definidas las principales entidades que se identifican en la actividad de una organización, además de mostrar las relaciones que existen entre ellas y los atributos de cada una, teniendo el cuidado especial de dar satisfacción a las necesidades de información de diversas áreas. Esta metodología ha favorecido la conceptualización de sistemas convergentes a este modelo corporativo que representa la realidad de la universidad en todos sus aspectos. El modelo, al igual que el plano regulador de una ciudad, establece los lineamientos de los futuros desarrollos y prevé necesidades de largo plazo, de manera tal que la infraestructura proyectada soporte el impacto de diferentes estilos de liderazgo y políticas al interior de la organización.

Con relación al Sistema Corporativo de la Universidad de Chile, uno de sus componentes que apoya más directamente los aspectos relativos a la docencia es el sistema GUIA ${ }^{12}$.

Sistema GUIA (Gestión universitaria de información al alumno)

Se orientó a satisfacer, de manera integral y bajo una visión de currículo transversal, las múltiples necesidades de información que tiene el estudiante de pregrado y posgrado de la institución, desde su ingreso hasta su titulación, incorporando aspectos académicos, socioeconómicos, culturales y financieros.

Para el estudiante, el producto se materializa en un portal personalizado, desde donde accede a información en todos los aspectos que le competen, permitiéndole interactuar con útiles servicios: registro de notas, inscripción de asignaturas, información de salas y horarios, encuestas de evaluación de cátedras, estado de cuenta corriente de aranceles, beneficios autorizados, crédito universitario, becas, entre otros.

12 El Sistema de Información Corporativo de la universidad incorpora otros componentes de apoyo a la gestión.

Ver sitio: http://www.sti.uchile.cl 


\section{DESAFÍOS FUTUROS}

Imaginarnos a la universidad del futuro es proyectarnos en una visión que tiene infinitas dimensiones, inclusive si lo restringimos al ámbito del software orientado a la docencia. Sin embargo, puedo compartir una visión que hemos desarrollado en uno de nuestros proyectos y que esperamos tenga un alto impacto en la enseñanza y en la búsqueda de nuevos paradigmas, que permitan entender y mejorar los procesos educativos, potenciando, además, la relación costo-efectividad en esta actividad.

El primer componente consiste en incorporar a la educación las prácticas hasta ahora desarrolladas para el e-business o comercio electrónico. Básicamente, se fundamenta en la oportunidad que existe hoy de identificar al estudiante, clasificarlo de acuerdo con un perfil determinado e inducirlo a seguir una ruta de navegación predefinida por el profesor y en función de las etapas o contenidos ya recorridos por este navegante-alumno. El modelo debe ser capaz de encontrar las relaciones temáticas del contenido que el alumno ha visitado y ofrecer, automáticamente, material relacionado, ya sea para su profundización o para su ampliación conceptual en otros contextos. Si adicionamos actividades evaluativas, conoceremos asimismo, su nivel de absorción de conocimiento.

Bajo este concepto, se tendrá información de los contenidos más visitados por los estudiantes, el nivel de aprendizaje y, simultáneamente, las distintas rutas seguidas por ellos. Este solo hecho permite al docente contrastar su estrategia pedagógica con los datos recogidos y potenciarla, o rediseñarla si fuese necesario, anticipándose a resultados definitivos. Ya en esta etapa habremos incorporado un instrumento poderosísimo que fortalece la relación profesor alumno en función de los objetivos de la clase.

Pero el desafío es mucho mayor: vivimos en un mundo donde disponemos de datos de todo tipo y en innumerables dimensiones. La dimensión social: nivel socioeconómico, región de origen, región de residencia, grupo etáreo; la académica: nivel de escolaridad, rendimiento promedio, puntajes de ingreso; la sicológica: preferencias, áreas de interés; la comercial, y muchas dimensiones más. Tenemos 
computadores que nos permiten combinar todos estos datos y estamos en condiciones de registrar el comportamiento de cada estudiante en su interacción con un medio educativo y de comunicación. Debemos, entonces, ser capaces de aprovechar esta oportunidad y lograr un cambio en la forma de incorporar la tecnología al proceso educativo.

El segundo paso es combinar todos estos datos con los resultados obtenidos en experiencias previas, establecer patrones de comportamiento, tipificar a los grupos de estudiantes que responden a cada patrón en función de todas las dimensiones disponibles y, sobre esta información, diseñar estrategias pedagógicas diferentes para cada uno de estos grupos. Estaremos ahora en presencia de un instrumento que nos permita diferenciar estadísticamente a nuestros alumnos y habremos dado un salto gravitante para aumentar la eficiencia de los programas.

Hasta esta etapa, el uso de la interactividad y de las técnicas estadísticas nos habrá permitido visualizar un enfoque empírico que orienta al profesor en la búsqueda de mejores prácticas educativas bajo un modelo previamente estructurado.

Pero las oportunidades continúan: podemos incorporar la inteligencia artificial para apoyarnos en el reconocimiento de los patrones de comportamiento, relacionarlos con la información individual del alumno y realizar predicciones sobre su rendimiento bajo el patrón que ha demostrado. De esta forma, el modelo deberá decidir automáticamente las medidas correctivas que deben adoptarse, induciendo al alumno por la vía de alertas y ofertas de contenidos atractivos o, simultáneamente, comunicando al profesor los hechos para emprender acciones por la vía de intervenciones directas.

Aplicando técnicas análogas, podemos encontrar y seguir los patrones de comportamiento de los alumnos más exitosos para ofrecerlos a los demás de acuerdo a su perfil individual.

Lograremos, entonces, construir un modelo de educación personalizada, obteniendo un gran provecho de la tecnología. Las herramientas las tenemos hoy, tal vez el futuro nos entregue más y mejores oportunidades. Sin embargo, debemos enfocar nuestros 
esfuerzos no hacia replicar nuestras actuales prácticas automatizándolas sino, más bien, hacia nuevos usos para mejorar la esencia de nuestra misión. 
